\title{
Session 2: Autoimmunity
}

\author{
Monday 16 September 2002, Moderators: Y. Shoenfeld and K. Potter
}

\section{[14.15-14.35]}

Production of human monoclonal antibodies to desmoglein 3 from Pemphigus vulgaris patient Kailash C. Bhol and A. Razzaque Ahmed Harvard School of Dental Medicine, Boston, USA

Pemphigus vulgaris is a potentially fatal autoimmune mucocutaneous disease associated with production of IgG autoantibodies to desmoglein 3, a $130 \mathrm{kDa}$ epidermal protein. To further characterize the epitope(s) of Pemphigus vulgaris antigen we established two humanhuman hybridomas by fusion of the peripheral blood mononuclear cells with a human and mouse heterohybridoma. These hybridomas designated as Mab Dsg3:06 and Mab Dsg-3:10 are stable in culture and demonstrated yields of monoclonal antibodies specific for Pemphigus vulgaris.

Immunofluorescence, immunoblot, ELISA assays demonstrated that both the monoclonal antibodies bind to the intercellular cement substance and to $130 \mathrm{kDa}$ protein present in the skin and specifically binds to recombinant desmoglein 3 protein, but not to desmoglein 1 protein. The epitope mapping experiment using 12 peptides spanning the extracellular domain of Pemphigus vulgaris antigen demonstrated that both the antibodies recognized the Bos 6 peptide and are of IgG1 subclass in nature. Both the antibodies were non-pathogenic as demonstrated in vitro by their inability to produce acantholysis in normal human skin in organ culture or in vivo by the induction of disease in neonatal BALB/c mice. The relevance and value of these monoclonal antibodies in the pathogenesis of Pemphigus vulgaris is discussed.

\section{[14.35-14.55]}

The mosaic of autoimmunity: The role of environmental factors in autoimmune disease Yehuda Shoenfeld The depart Medicine B, Research Center for Autoimmunity Sheba Medical Center, Tel aviv Univesity, Israel

E-mail: shoenfel@post.tau.ac.il
Autoimmune diseases are conditions in which the immune system damages normal components of the individual. Initially it was thought that autoimmune disease was the inevitable outcome of the presence of clones of lymphocytes with receptors that recognize self-antigens. Thus tolerance to self, the state of non-autoimmunity, was due to the absence of selfrecognizing lymphocytes, the 'forbidden' culprits of autoimmune disease. Autoimmune diseases were found to be multifactorial in their etiology. For practical reasons these factors are classified into four categories:

Genetic: which entail the MHC class I, II, and III. A case in point will be the haplotypes of HLA-DR3, B8 which are prevalent in many classical diseases. In addition $\mathrm{Gm}$ allotype, idiotypes and some complement deficiencies may have a genetic background.

Immune deficiencies: $\mathrm{C} 1 \mathrm{q} \mathrm{C} 2, \mathrm{C} 4$ and $\mathrm{IgA}$ deficiencies are among the most common defects associated with diverse autoimmune conditions.

Hormonal state: most autoimmune diseases are detected in females at the child bearing ages. The role of estrogens will be delineated. In addition other hormones play a role i.e. prolactin.

Environmental causes: Those are the most important as a trigger factors determining the time and type of disease. They entail infectious agents, chemicals, drugs and even vaccines.

The type of disease in an individual, in an autoimmune prone family, will be determined by the specific combination of the different factors mentioned above.

A special case report of a 45 years old lady (HLA DR3, B8) who developed 8 autoimmune diseases and 10 different autoantibodies, upon accidental exposure to a chemical, compound (a polyclonal activator), will be detailed.

\section{Reference}

[1] Y. Shoenfeld and D.A. Isenberg, The Mosaic of Autoimmunity. (English), Elsevier, Holland, 1989, pp. 400.

[2] Y. Shoenfeld and A. Aron-Maor, Vaccination and autoimmu- 
nity - 'Vaccinosis': a dangerous liaison? J Autoimmunity 14 (2000), 1-10.

[3] D. Rahamim-Cohen and Y. Shoenfeld, The mosaic of autoimmunity. A classical case of inhalation of a polyclonal activating factor in a genetically and hormonally susceptible patient leading to multiple autoimmune diseases, IMAJ 3 (2001), 381-382.

[14.55-15.15]

Similar patters of B cell perturbation are found in the peripheral blood from patients with systemic lupus erythematosis (SLE) and infectious mononucleosis (IM)

K.N. Potter, I. Mockridge, A. Rahman, S. Buchan, T. Hamblin, D.A. Isenberg and F.K. Stevenson Tenovus Labortaory, University of Southampton, UK

Both systemic lupus erythematosus (SLE) patients with active disease and individuals with bacterial infections exhibit B cell lymphopenia combined with an increased frequency of plasmablast/early plasma cells in the peripheral blood. These data suggested that the disturbance in immune system in SLE patients is similar to that which occurs during infectious disease. One characteristic of SLE is the selective rise in level of V4-34 encoded immunoglobulins. Infectious mononucleosis (IM), which is caused by infection with Epstein-Barr virus, is also characterized by the selective rise in V4-34 encoded immunoglobulins. Our objectives were to determine whether the evidence for antigen stimulation in SLE paralleled those found in IM to understand the nature of the immune activation in SLE. We compared the distribution of B cell subpopulations in the peripheral blood of patients with active and inactive SLE with that found during IM. We found parallels in the B cell population profiles between SLE and IM. The early plasma cell number is maintained at healthy levels in both active and inactive SLE, and increased in IM despite an overall B cell lymphopenia involving both naive and memory B cells in both diseases. Evidence of clonality and intraclonal variation was found in V4-34 encoded immunoglobulins isolated from the early plasma cells from both SLE and IM patients. Both sets of V4-34 encoded IgG generally were mutated, with some clones being unmutated IgG. These results suggest a chronic stimulation of the immune system in SLE by an antigen which can stimulate V4-34 Ig production. One possibility includes reactivation of endogenous herpes viruses due to immunosuppression.
[15.45-16.05]

Search for a monoclonal antibody mimicking naturally occurring anti-C3/C3b antibodies that regulate complement amplification

Hans U. Lutz ${ }^{\mathrm{a}}$, Markus Schlumberger ${ }^{\mathrm{a}}$, Peter J. Späth $^{\mathrm{b}}$, Emiliana Jelezarova ${ }^{\mathrm{a}}$ and Alois B. Lang ${ }^{\mathrm{c}}$ a Institute of Biochemistry, Swiss Federal Institute of Technology, ETH-Hönggerberg, HPM, CH 8093 Zurich, Switzerland

${ }^{\mathrm{b}}$ ZLB Bioplasma Ltd., Berne, Switzerland

${ }^{\mathrm{c}}$ Department of Immunology, Berna Biotech Ltd., Berne, Switzerland

Whole human IgG (IVIG) has a complement attenuating effect by stimulating the physiological inactivation of the most effective precursor of the complement amplifying C3 convertase in vitro (Lutz et al., Blood. 1996;88:184-193). The most effective C3 convertase precursors generated in human plasma contain $\mathrm{C} 3 \mathrm{~b}$ in dimeric form with $\mathrm{C}_{3} \mathrm{~b}_{2}$-IgG complexes representing the predominant species (Jelezarova et al., Biochem. J. 2000; 349: 217-223). IVIG has anti-inflammatory effects in several complement-dependent autoimmune diseases (Dalakas, Muscle Nerve. 1999; 22: 14791497). IVIG applied at $2 \mathrm{~g} / \mathrm{kg}$ body weight attenuated complement amplification in dermatomyositis and indeed stimulated inactivation of C3bn-containing complexes in vivo (Lutz, Stammler, Bianchi, Trueb, Hunziker, Burger, Jelezarova and Späth, in preparation). It attenuated C3 consumption in myopathic and amyopathic dermatomyositis, but did not inhibit classical pathway activation. This regulatory, anti-inflammatory principle appears to be enriched in naturally occurring IgG anti-C3/C3b antibodies (anti-C3 NAbs) which are present in IVIG. F(ab') $)_{2}$ fragments of anti-C3 NAbs, affinity purified from IVIG, exerted a complement attenuating effect already when added at $1-20 \mu \mathrm{g} / \mathrm{ml}$ to $20 \%$ serum in which complement was activated by an immune aggregate. These NAbs differ from autoaggressive antibodies called $\mathrm{C} 3$ nephritic factors $(\mathrm{C} 3 \mathrm{NeF})$ that stabilize $\mathrm{C} 3$ convertase and augment $\mathrm{C} 3$ consumption. To investigate and exploit the presumed regulatory potency of anti-C3 antibodies we generate human monoclonal anti-C3 antibodies and study their capacity to counteract $\mathrm{C} 3 \mathrm{NeF}$ in an in vitro assay system (Jelezarova et al., J. Immunol. Meth. 2001;251:45-52) and their ability to stimulate inactivation of $\mathrm{C} 3$ convertase precursors. 
[16.05-16.25]

Comparability testing of a recombinant human anti-Rh $D$ antibody derived from two different cell lines using biophysical and biochemical parameters: Relevance for scale up manufacturing Hans-Joachim Krieg ${ }^{\mathrm{a}}$, Stefan Schürch ${ }^{\mathrm{b}}$, Johann Schaller $^{\mathrm{b}}$, Sébastien Déjardin ${ }^{\mathrm{a}}, \mathrm{Anh} \mathrm{Tu}^{\mathrm{a}}$, Sylvia Miescher $^{\mathrm{a}, \mathrm{c}}$, Pierre-Alain Girod ${ }^{\mathrm{d}}$, Nicolas Mermod ${ }^{\mathrm{d}}$ and Hanspeter Amstutz

${ }^{\mathrm{a}} Z L B$ Bioplasma AG, Bern, Switzerland

${ }^{\mathrm{b}}$ Department Chemistry and Biochemistry, University of Bern, Switzerland

${ }^{\mathrm{c}}$ Institute of Immunology, University of Bern, Switzerland

${ }^{\mathrm{d}}$ Laboratory of Molecular Biotechnology, University of Lausanne, Switzerland

A recombinant anti-Rh $\mathrm{D}$ antibody, originally isolated from a hyperimmune combinatorial antibody library has been expressed as a full length IgG1 in a variety of eucaryotic production systems including SP2/0, HEK and CHO cells. A CHO cell line, MDJ8S, which shows excellent long term stability characteristics has been established with a view to market production of the anti-Rh D antibody. Additionally, in order to further increase production rates, matrix attachment region (MAR) elements were successfully introduced resulting in a second generation $\mathrm{CHO}$ cell line, AMW1 which shows a log-fold increase in secreted antibody.

To demonstrate comparability of the anti-Rh D antibody derived from the two cell lines a variety of biochemical and functional tests were performed to assess the microheterogeneity and biological activity of the product. Results from biochemical and biophysical (SDS-PAGE, size exclusion chromatography, IEF, oligosaccharide profile analysis, tryptic peptide mapping, MS, MS/MS) analyses as well as from biological assays (FACS analysis, phagocytosis and ADCC) will be discussed.

\section{[16.25-16.45]}

ANTI-dsDNA anti-idiotypic activity as the main mechanism of IVIG effect in SLE

L. Rauova ${ }^{\mathrm{a}, \mathrm{c}}$, B. Gilburd ${ }^{\mathrm{a}}$, M. Blank ${ }^{\mathrm{a}}$, I. Goldberg ${ }^{\mathrm{b}}$, J. Kopolovic ${ }^{\mathrm{b}}$, M. Ehrenfeld ${ }^{\mathrm{a}}$, J. Rovensky ${ }^{\mathrm{c}}$ and Y. Shoenfeld ${ }^{\mathrm{a}}$

${ }^{\text {a } C e n t e r ~ f o r ~ A u t o i m m u n e ~ D i s e a s e s, ~ D e p a r t m e n t ~ o f ~}$ Internal Medicine B, Sheba Medical Center, Tel Hashomer, Israel

${ }^{\mathrm{b}}$ Department of Pathology, Sheba Medical Center, Tel Hashomer, Israel

${ }^{\mathrm{c}}$ Institute of Rheumatic Diseases, Piestany, Slovakia
Anti-idiotypic modulation by concentrated specific natural polyclonal anti-dsDNA anti-idiotypic antibodies affinity purified from IVIG was employed in the treatment of experimental SLE.

Methods: Specific natural polyclonal anti-dsDNA anti-idiotypic antibodies (IVIG-ID) were affinity purified from IVIG on an anti-dsDNA-Sepharose column constructed with anti-dsDNA idiotypes affinity purified from 55 patients with SLE. The NZB/W F1 mice were treated with 3 weekly intravenous injections of IVIGID (2 mg/kg/inj) and regular IVIG (400 mg/kg/inj) at the age of 21 weeks (after developing of anti-dsDNA antibodies) and 8 weeks, respectively.

Results: The IVIG-ID treated mice showed a decline in the titer of a-dsDNA antibodies during the treatment, reaching maximum effect one week after the last injection. This effect of treatment sustained as long as 2 month after completing the treatment. The incidence of proteinuria was significantly lower in IVIG-ID treated mice compared to non treated ones. The treatment with IVIG-ID at the age of 21 weeks capable to prevent the progression of IgG deposits in the glomerular basement membrane without affecting the mesangial deposits. An early treatment with IVIG-ID and with high dose IVIG at the age of 8 weeks resulted also in retardation of the kidney mesangial deposits.

Conclusion: Treatment with concentrated specific anti-dsDNA-anti-ID prepared from commercial IVIG is more effective in suppression of humoral and clinical signs of SLE than regular IVIG. In agreement with the network theory, these results point to the considerable regulatory role of anti-idiotypes in the mechanism of action of IVIG in SLE.

\section{[16.45-17.05]}

\section{Functional properties of anti-D monoclonal} antibodies: Role of host cell expression system and in vitro correlation between ADCC and IL2 release from Jurkat CD16

C. De Romeuf, R. Beliard, C. Gaucher, A. Glacet and D. Bourel

Research Department. LFB, 59 rue de Trévise. 59011, Lille cedex, France

Many human monoclonal Rh-D antibodies have already been produced in the last decade. The mechanism which leads to the absence of foeto-maternal alloimmunization is not totally understood. However, one of the admitted "in vivo" properties of polyclonal antibodies is the ability to eliminate Rhesus positive red blood cells (RBC) from the circulation. 
In this study, three monoclonal antibodies (Mab) anti-D were compared. -Mab DF5-EBV was produced by human B cell, obtained from immunized D-negative donor and immortalized by Epstein-Barr virus transformation. This Mab was used as a negative control since it was found unable to eliminate Rhesus positive $\mathrm{RBC}$ from the circulation in a previous clinical trial. -Mab named DF5-YB2/0, was obtained by expressing the primary sequence of the DF5-EBV in YB2/0 cell line. -Mab R297, an other recombinant antibody, was also expressed in YB2/0 cell line.

In the first experiment, these Mabs were evaluated "in vitro" for their ability to induce the lysis of papaine treated RBC using PBL as effector cells. All assays were performed in the presence of human immunoglobulins (IVIg) in order to reconstitute the physiological conditions. IVIg are presumed to bind to the high affinity Fc gamma RI (CD64). Both Mab R297 and Mab DF5-YB2/0 induced RBC lysis in an extent comparable to that obtained with the Polyclonal antibodies WinRho whereas DF5-EBV was totally ineffective.

In the second experiment, purified NK cells and untreated RBC were used as effector cells and target cells, respectively. After 5 hours incubation time, Mabs antiD R297 and DF5-YB2/0 but not DF5-EBV were able to support RBC lysis.

In both experiments, red cell lysis was inhibited by Mab 3G8 directed against the Fc $\gamma$ RIII (CD16).

Taken together, these results demonstrated that ADCC mediated by Mab R297 as well as Mab DF5YB2/0 involved Fc $\gamma$ RIII expressed on NK cells.

In an attempt to find another "in vitro" assay to evaluate Mab anti-D, a third experiment was performed to assess the ability of these Mabs to activate Jurkat CD16 cell line. For that, Mab were incubated overnight with Rhesus positive red cells and Jurkat CD16, then IL2 released in the supernatant was measured by ELISA. A strong correlation between the ADCC and the activation of Jurkat CD16 was observed, suggesting that this assay might be used to discriminate Mabs anti-D for their reactivity toward Fc $\gamma$ RIII (CD16).

In conclusion, these data pointed out that posttraductional events more than aminoacid sequence are crucial to support an Fc $\gamma$ RIII (CD16) specific ADCC activity and that release of IL2 from Jurkat CD16 cells might reflect this activity.

[17.05-17.25]

In vitro functional assessment of a recombinant anti-Rh D antibody: Relevance to clinical performance
Hanspeter Amstutz ${ }^{\mathrm{a}}$, Sébastien Déjardin ${ }^{\mathrm{a}}$, Thomas Iff $^{\mathrm{a}}$, Martin Spycher ${ }^{\mathrm{a}}$, Andreas Hofmann ${ }^{\mathrm{b}}$, Stefan Nahrgang $^{\mathrm{c}}$, Beda Stadler ${ }^{\mathrm{b}}$, Sylvia Miescher ${ }^{\mathrm{a}, \mathrm{b}}$ and Peter Lerch ${ }^{\mathrm{a}}$

${ }^{a} Z L B$ Bioplasma AG, Bern, Switzerland

${ }^{\mathrm{b}}$ Institute of Immunology, University of Bern, Switzerland

${ }^{\mathrm{c}}$ Department Genie Chimique, EPFL, Lausanne, Switzerland

A recombinant human anti-Rh $\mathrm{D}$ antibody has been produced by transfecting the relevant Fab (originally derived from a hyperimmune phage library) and $\mathrm{Fc}$ $(\mathrm{CH} \gamma 1, \mathrm{C} \kappa)$ gene fragments into $\mathrm{CHO}$ DG 44 cells. Successive cloning and selection steps resulted in a stable cell line, MDJ8s as previously described (Miescher et al, BrJHaemat., 2000). The resulting $\operatorname{IgG} 1 \kappa$ anti-Rh D antibody is projected to replace the hyperimmune polyclonal immunoglobulin products currently used to prevent haemolytic disease of the newborn (HDN) caused by Rhesus incompatibility. Before going to clinical trial a full functional analysis of the anti$\mathrm{Rh} \mathrm{D}$ antibody must be performed. In this respect, two major difficulties must be acknowledged. Firstly, there is no feasible animal model of $\mathrm{Rh}$ incompatibility and secondly, the mechanism of Rh prophylaxis, whilst in use for many years is not understood. This complicates the design of functional assays as they cannot be targeted to functional attributes of a successful antibody for Rhesus prophylaxis. As current opinion indicates that both intact Fab and Fc functions are required for efficacy we employed in vitro functional tests aimed at the individual Fab and Fc parts as well as the whole IgG1 molecule.

Such tests include FACS analysis of Fab binding to $\mathrm{RhD}$ positive rbc and Fc receptor binding as well as biological assays measuring phagocytosis by chemiluminescence (CLT), rosette formation and lysis of rbc by antibody dependent cellular cytotoxicity (ADCC).

The Fab binding to rbc indicates a high affinity and specific antibody. In contrast, Fc functional tests which use human effector cells are susceptible to considerable variation caused by different donors, subpopulations of cells tested, antibody concentrations, presence/absence of inhibitory factors such that interpretation and comparison with other data in the literature is not evident. Above all the major question which will be discussed remains the relevance of these in vitro tests for predicting clinical efficacy. 